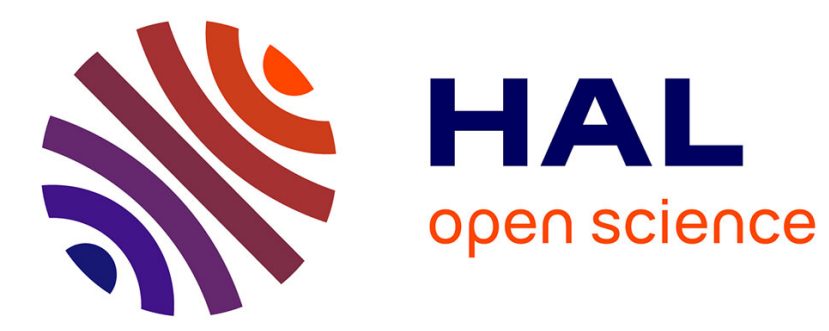

\title{
Single output dependent quadratic observability normal form
}

\author{
Gang Zheng, Driss Boutat, Jean-Pierre Barbot
}

\section{To cite this version:}

Gang Zheng, Driss Boutat, Jean-Pierre Barbot. Single output dependent quadratic observability normal form. NOLCOS 2007 - 7th IFAC Symposium on Nonlinear Control Systems, Aug 2007, Pretoria, South Africa. pp.432-437, 10.3182/20070822-3-ZA-2920.00071 . inria-00179222

\section{HAL Id: inria-00179222 \\ https://hal.inria.fr/inria-00179222}

Submitted on 14 Oct 2007

HAL is a multi-disciplinary open access archive for the deposit and dissemination of scientific research documents, whether they are published or not. The documents may come from teaching and research institutions in France or abroad, or from public or private research centers.
L'archive ouverte pluridisciplinaire HAL, est destinée au dépôt et à la diffusion de documents scientifiques de niveau recherche, publiés ou non, émanant des établissements d'enseignement et de recherche français ou étrangers, des laboratoires publics ou privés. 


\title{
SINGLE OUTPUT DEPENDENT QUADRATIC OBSERVABILITY NORMAL FORM
}

\author{
G. Zheng* D. Boutat ${ }^{* *}$ J.P. Barbot ${ }^{* * *}$
}

\author{
* INRIA Rhône-Alpes, Inovallée, 655 avenue de l'Europe, \\ Montbonnot Saint Martin, 38334 St Ismier Cedex, France \\ ** LVR/ENSI, 10 Boulevard de Lahitolle, 18020 Bourges, \\ France \\ *** ECS/ENSEA, 6 Avenue du Ponceau, 95014 \\ Cergy-Pontoise, France, Projet ALIEN-INRIA Futures
}

\begin{abstract}
In this paper, two quadratic observability normal forms dependent on output respectively correspondent to drift dominant term and forced dominant term are firstly discussed. Moreover characteristic numbers are studied in order to simplify the calculation of these two normal forms.
\end{abstract}

\section{INTRODUCTION}

Normal form is a powerful tool to analyze the properties of dynamical systems, such as stability (Poincaré et al., 1899), controllability (Kang et al., 1992). In 2001, this concept was firstly introduced in (Boutat-Baddas et al., 2001) in order to analyze the observability of dynamical system, in which the quadratic observability normal form was studied and the interests of this technique was highlighted. In 2005, a new observability normal form dependent on its output was studied in (Zheng et al., 2005), and the necessary and sufficient conditions were proposed. Moreover, an extension to multi-outputs case has also been analyzed in (Boutat et al., 2006). Since the observability normal form dependent on its output (or outputs) were exactly linear, another reasonable extension based on the work of (Boutat-Baddas et al., 2001) and (Chabraoui et al., 2003) is the study of quadratic observability normal form dependent on its output (or outputs).

Therefore, in this paper, we consider the following single input single output system:

$$
\left\{\begin{array}{l}
\dot{\zeta}=f(\zeta)+g(\zeta) u \\
y=h(\zeta)
\end{array}\right.
$$

where $\zeta \in D \subset \mathbb{R}^{n}, u \in \mathbb{R}, f: \mathbb{R}^{n} \rightarrow \mathbb{R}^{n}$, $g: \mathbb{R}^{n} \rightarrow \mathbb{R}^{n}$ and $h: \mathbb{R}^{n} \rightarrow \mathbb{R}$ are analytic functions, and assume that for all $\zeta \in D$, we have $\operatorname{rank}\left[d h, d L_{f} h, \ldots, d L_{f}^{n-1} h\right]^{T}=n$.

As an extension of our work in (Zheng et al., 2005), if the theorem in (Zheng et al., 2005) is not verified, we assume that system (1) can be transformed into the following form ${ }^{1}$ :

$$
\left\{\begin{array}{c}
\dot{\eta}=\beta(y)+A(y) \eta+\gamma_{y}^{[2]}(\bar{\eta}) \\
\quad+B(y) u+\vartheta_{y}^{[1]}(\bar{\eta}) u+O_{y}^{[3]}(\bar{\eta}, u) \\
y=C \eta
\end{array}\right.
$$

where $\eta \in \mathbb{R}^{n}, \bar{\eta}=\left[\eta_{1}, \ldots \eta_{n-1}\right]^{T}$,

$$
\begin{aligned}
\gamma_{y}^{[2]}(\bar{\eta}) & =\left[\gamma_{y_{1}}^{[2]}(\bar{\eta}), \ldots, \gamma_{y_{n}}^{[2]}(\bar{\eta})\right]^{T}, \\
\vartheta_{y}^{[1]}(\bar{\eta}) & =\left[\vartheta_{y_{1}}^{[1]}(\bar{\eta}), \ldots, \vartheta_{y_{n}}^{[1]}(\bar{\eta})\right]^{T},
\end{aligned}
$$

and for $1 \leq i \leq n, \gamma_{y_{i}}^{[2]}(\bar{\eta})$ and $\vartheta_{y_{i}}^{[1]}(\bar{\eta})$ are function of $\bar{\eta}$ with order 2 and 1 respectively parameterized by $y, \beta(y)=\left[\beta_{1}(y), \ldots, \beta_{n}(y)\right]^{T}$, and

$$
A(y)=\left(\begin{array}{cccc}
0 & \ldots & 0 & 0 \\
\alpha_{1}(y) & \cdots & 0 & 0 \\
\vdots & \ddots & \ldots & \vdots \\
0 & 0 & \alpha_{n-1}(y) & 0
\end{array}\right),
$$

\footnotetext{
1 The necessary and sufficient condition for this transformation is still an open question.
} 
and $B(y)$ is defined as follows:

$$
B(y)=(b(y), 0, \cdots, 0)^{T}
$$

As it was shown in (Boutat-Baddas et al., 2001) that the equivalence modulo an output injection is justified by the fact that the output injection can be canceled in the observation error dynamics. Therefore, by an output injection $\beta(y)$, system (2) is equivalent modulo an output injection to the following system

$$
\left\{\begin{array}{l}
\dot{x}=A(y) x+\gamma_{y}^{[2]}(\bar{x}) \\
\quad+B(y) u+\vartheta_{y}^{[1]}(\bar{x}) u+O_{y}^{[3]}(\bar{x}, u) \\
y=x_{n}=C x
\end{array}\right.
$$

where $\bar{x}=\left[x_{1}, \ldots, x_{n-1}\right]^{T}$.

Our problem is how to characterize the fact that all the quadratic terms can be canceled by a diffeomorphism? If this kind of diffeomorphism does not exist, then what's its normal form and its resonant terms?

\section{QUADRATIC EQUIVALENCE MODULO AN OUTPUT INJECTION}

Definition 1. System (4) is quadratically equivalent to system

$$
\left\{\begin{array}{l}
\dot{z}=A(y) z+\tilde{\gamma}_{y}^{[2]}(\bar{z}) \\
\quad+B(y) u+\bar{\vartheta}_{y}^{[1]}(\bar{z}) u+O_{y}^{[3]}(\bar{z}, u) \\
y=C z
\end{array}\right.
$$

if there exists a diffeomorphism of the following form:

$$
z=x+\phi_{y}^{[2]}(\bar{x})
$$

which transforms the quadratic term $\gamma_{y}^{[2]}(\bar{x})$ into another quadratic term $\tilde{\gamma}_{y}^{[2]}(\bar{z})$, where $\phi_{y}^{[2]}(\bar{x})=$ $\left[\phi_{y, 1}^{[2]}(\bar{x}), \ldots, \phi_{y, n}^{[2]}(\bar{x})\right]^{T}$ and $\phi_{y, i}^{[2]}(\bar{x})$ are the homogenous polynomials with order 2 in $z$.

Remark 1. i) In order to keep the output unchanged, we choose the output equal to $x_{n}$, which means the diffeomorphism $z=x+\phi_{y}^{[2]}(\bar{x})$ should verify $\phi_{y, n}^{[2]}(\bar{x})=0$.

ii) It should be noted that this choice is not obligatory. In fact, we can also choose $\phi_{y, n}^{[2]}(\bar{x})=$ $\phi_{y, n}^{[0]}(y)$, i.e., a function of the output.

Proposition 1. System (4) is quadratically equivalent to system (5) modulo an output injection, if and only if the following homologic equations are satisfied:

$$
\left\{\begin{array}{l}
\gamma_{y}^{[2]}(\bar{x})+\Gamma_{y}^{[2]}(\bar{x})=A(y) \phi_{y}^{[2]}(\bar{x})+\tilde{\gamma}_{y}^{[2]}(\bar{x}) \\
\vartheta_{y}^{[1]}(\bar{x})+\frac{\partial \phi_{y}^{[2]}(\bar{x})}{\partial x_{1}} b(y)=\bar{\vartheta}_{y}^{[1]}(\bar{x})
\end{array}\right.
$$

where

$$
\begin{aligned}
& \Gamma_{y}^{[2]}(\bar{x})= \\
& {\left[\frac{\partial \phi_{y}^{[2]}(\bar{x})}{\partial x_{2}} \alpha_{1}(y), \cdots, \frac{\partial \phi_{y}^{[2]}(\bar{x})}{\partial x_{n-1}} \alpha_{n-2}(y), 0,0\right] x .}
\end{aligned}
$$

Assume that $z=x+\phi_{y}^{[2]}(\bar{x})$, hence $\dot{z}=\dot{x}+\frac{\partial \phi_{y}^{[2]}(\bar{x})}{\partial x} \dot{x}$

According to equation (4) and (5), we have

$$
\begin{aligned}
\dot{z}= & {\left[1+\frac{\partial \phi_{y}^{[2]}(\bar{x})}{\partial x}\right]\left[\begin{array}{c}
A(y) x+\gamma_{y}^{[2]}(\bar{x})+B(y) u \\
+\vartheta_{y}^{[1]}(\bar{x}) u+O_{y}^{[3]}(\bar{x}, u)
\end{array}\right] } \\
= & A(y)\left(x+\phi_{y}^{[2]}(\bar{x})\right)+\tilde{\gamma}_{y}^{[2]}\left(\bar{x}+\phi_{y}^{[2]}(\bar{x})\right) \\
& +B(y) u+\bar{\vartheta}_{y}^{[1]}(\bar{x}) u+O_{y}^{[3]}(\bar{x}, u)
\end{aligned}
$$

So we obtain

$$
\left\{\begin{array}{l}
\gamma_{y}^{[2]}(\bar{x})+\frac{\partial \phi_{y}^{[2]}(\bar{x})}{\partial x} A(y) x=A(y) \phi_{y}^{[2]}(\bar{x})+\tilde{\gamma}_{y}^{[2]}(\bar{x}) \\
\vartheta_{y}^{[1]}(\bar{x})+\frac{\partial \phi_{y}^{[2]}(\bar{x})}{\partial x} B(y)=\bar{\vartheta}_{y}^{[1]}(\bar{x})
\end{array}\right.
$$

where

$$
\begin{aligned}
& \frac{\partial \phi_{y}^{[2]}(\bar{x})}{\partial x} A(y) x \\
& =\left[\frac{\partial \phi_{y}^{[2]}(\bar{x})}{\partial x_{2}} \alpha_{1}(y), \cdots, \frac{\partial \phi_{y}^{[2]}(\bar{x})}{\partial y} \alpha_{n-1}(y), 0,0\right] x \\
& =\Gamma_{y}^{[2]}(\bar{x})+O_{y}^{[3]}(\bar{x})
\end{aligned}
$$

and finally equation (8) becomes:

$$
\left\{\begin{array}{l}
\gamma_{y}^{[2]}(\bar{x})+\Gamma_{y}^{[2]}(\bar{x})=A(y) \phi_{y}^{[2]}(\bar{x})+\tilde{\gamma}_{y}^{[2]}(\bar{x}) \\
\vartheta_{y}^{[1]}(\bar{x})+\frac{\partial \phi_{y}^{[2]}(\bar{x})}{\partial x_{1}} b(y)=\bar{\vartheta}_{y}^{[1]}(\bar{x}) .
\end{array}\right.
$$

\section{QUADRATIC OBSERVABILITY NORMAL FORM DEPENDENT ON ITS OUTPUT}

Since there exist two quadratic terms in system (5): $\tilde{\gamma}_{y}^{[2]}(\bar{z})$ and $\bar{\vartheta}_{y}^{[1]}(\bar{z}) u$, we will study two normal form which correspond respectively to the drift term and forced term in this section.

\subsection{Normal form with drift dominant term}

In this subsection, we study the normal form correspondent to drift dominant term by simplifying the quadratic term $\tilde{\gamma}_{y}^{[2]}(\bar{z})$.

Theorem 1. Normal form correspondent to drift dominant term system (5) by quadratically equivalent modulo an output injection is in the following normal form:




Since the objective of this normal form is to make $\tilde{\gamma}_{y}^{[2]}(\bar{x})=0$, so the first homologic equation in (7) becomes

$$
\gamma_{y}^{[2]}(\bar{x})+\Gamma_{y}^{[2]}(\bar{x})=A(y) \phi_{y}^{[2]}(\bar{x})
$$

Define $\left\{\begin{array}{l}\phi_{y}^{[2]}(\bar{x})=\left[\phi_{y, 1}^{[2]}(\bar{x}), \ldots, \phi_{y, n}^{[2]}(\bar{x})\right]^{T}, \\ \gamma_{y}^{[2]}(\bar{x})=\left[\gamma_{y, 1}^{[2]}(\bar{x}), \ldots, \gamma_{y, n}^{[2]}(\bar{x})\right]^{T}, \\ \vartheta_{y}^{[1]}(\bar{x})=\left[\vartheta_{y, 1}^{[1]}(\bar{x}), \ldots, \vartheta_{y, n}^{[1]}(\bar{x})\right]^{T},\end{array}\right.$ where

$$
\phi_{y_{n}}^{[2]}(\bar{x})=0 .
$$

And we obtain

$$
\left\{\begin{array}{c}
\alpha_{1}(y) \phi_{y, 1}^{[2]}(\bar{x})=\gamma_{y, 2}^{[2]}(\bar{x})+\sum_{i=1}^{n-2}\left[\frac{\partial \phi_{y, 2}^{[2]}(\bar{x})}{\partial x_{i+1}} \alpha_{i}(y) x_{i}\right] \\
\vdots \\
\alpha_{n-2}(y) \phi_{y, n-2}^{[2]}(\bar{x})=\gamma_{y, n-1}^{[2]}(\bar{x}) \\
+\sum_{i=1}^{n-2}\left[\frac{\partial \phi_{y, n-1}^{[2]}(\bar{x})}{\partial x_{i+1}} \alpha_{i}(y) x_{i}\right] \\
\alpha_{n-1}(y) \phi_{y, n-1}^{[2]}(\bar{x})=\gamma_{y, n}^{[2]}(\bar{x})
\end{array}\right.
$$

and the first line of equation (7) gives

$$
\gamma_{y, 1}^{[2]}(\bar{x})+\sum_{i=1}^{n-2}\left[\frac{\partial \phi_{y, 1}^{[2]}(\bar{x})}{\partial x_{i+1}} x_{i} \alpha_{i}(y)\right]=0
$$

Equation (10) can be used to deduce $\phi_{y, i}^{[2]}(\bar{x})$ in order to cancel the quadratic terms from $\gamma_{y, 2}^{[2]}(\bar{x})$ to $\gamma_{y, n}^{[2]}(\bar{x})$ respectively. Moreover, if $\gamma_{y, i}^{[2]}(\bar{x})$ and $\phi_{y, i}^{[2]}(\bar{x})$ verify also equation (11), then this system can be quadratically linearizable. Otherwise, it gives the following resonant terms:

$$
\gamma_{y, 1}^{[2]}(\bar{x})+\sum_{i=1}^{n-2} \alpha_{i}(y) \frac{\partial \phi_{y, 1}^{[2]}(\bar{x})}{\partial x_{i+1}} x_{i} .
$$

With $\vartheta_{y}^{[1]}(\bar{x})+\frac{\partial \phi_{y}^{[2]}(\bar{x})}{\partial x_{1}} b(y)=\bar{\vartheta}_{y}^{[1]}(\bar{x})$, we have $\bar{\vartheta}_{y, n}^{[1]}(\bar{x})=$ $\vartheta_{y, n}^{[1]}(\bar{x})$. Assume $\bar{\vartheta}_{y, n}^{[1]}(\bar{x})=\sum_{i=1}^{n-1} \bar{c}_{i}^{n}(y) x_{i}$ and $\vartheta_{y, n}^{[1]}(\bar{x})=$ $\sum_{i=1}^{n-1} c_{i}^{n}(y) x_{i}$, then $\bar{c}_{i}^{n}(y)=c_{i}^{n}(y)$. And it is not possible to cancel other components. Hence we obtain normal form (9).

In order to highlight the proposed method, we consider the following example.

Example 1. Consider the following system:

$$
\left\{\begin{aligned}
& \dot{x}_{1}=\left(x_{1}^{2}-2 x_{3}^{2} x_{1} x_{2}+x_{3}^{2} x_{2}^{2}\right) \\
& \quad+\left(x_{3}-4 x_{3}^{2} x_{1}-2 x_{3}^{4} x_{2}\right) u \\
& \dot{x}_{2}= x_{3} x_{1}+x_{3}^{2} x_{2}^{2}-\left(x_{3} x_{2}+2 x_{3}^{2} x_{1}\right) u \\
& \dot{x}_{3}=x_{3} x_{2}+x_{3}^{2} x_{1} u \\
& y=x_{3}
\end{aligned}\right.
$$

where $y \neq 0$. According to equation (10), we have

$$
\left\{\begin{array}{l}
\phi_{y, 3}^{[2]}(\bar{x})=0 \\
\alpha_{2}(y) \phi_{y, 2}^{[2]}(\bar{x})=\gamma_{y, 3}^{[2]}(\bar{x}) \\
\alpha_{1}(y) \phi_{y, 1}^{[2]}(\bar{x})=\gamma_{y, 2}^{[2]}(\bar{x})+\alpha_{1}(y) \frac{\partial \phi_{y, 2}^{[2]}(\bar{x})}{\partial x_{2}} x_{1}
\end{array}\right.
$$

and we obtain $\left\{\begin{array}{l}z_{1}=x_{1}+x_{3} x_{2}^{2} \\ z_{2}=x_{2} \\ z_{3}=x_{3}\end{array}\right\}$. Hence the quadratic resonant terms are:

$$
\gamma_{y, 1}^{[2]}(\bar{x})+\alpha_{1}(y) \frac{\partial \phi_{y, 1}^{[2]}(\bar{x})}{\partial x_{2}} x_{1}=x_{1}^{2}+x_{3}^{2} x_{2}^{2}
$$

Finally we have the following normal form:

$$
\left\{\begin{array}{l}
\dot{z}_{1}=z_{1}^{2}+z_{3}^{2} z_{2}^{2}+\left(z_{3}-4 z_{3}^{2} z_{1}-2 z_{3}^{4} z_{2}\right) u \\
\quad+O_{y}^{[3]}\left(z_{1}, z_{2}, u\right) \\
\dot{z}_{2}=z_{3} z_{1}-\left(z_{3} z_{2}+2 z_{3}^{2} z_{1}\right) u+O_{y}^{[3]}\left(z_{1}, z_{2}, u\right) \\
\dot{z}_{3}=z_{3} z_{2}+z_{3}^{2} z_{1} u+O_{y}^{[3]}\left(z_{1}, z_{2}, u\right) \\
y=z_{3}
\end{array}\right.
$$

\subsection{Normal form with forced dominant term}

This subsection is devoted to study another normal form by simplifying quadratic terms: $\bar{\vartheta}_{y}^{[1]}(\bar{z}) u$, in system (5).

Theorem 2. Normal form forced dominant term of system (5) by quadratically equivalent modulo an output injection is in the following form:

$$
\left\{\begin{array}{c}
\dot{\xi}=\left(\begin{array}{c}
\sum_{j \geq i=1}^{n-1} d_{i}^{1}(y) \xi_{i} \xi_{j} \\
\xi_{1} \sum_{i=1}^{n-1} d_{i}^{2}(y) \xi_{i} \\
\vdots \\
\xi_{1} \sum_{i=1}^{n-1} d_{i}^{n}(y) \xi_{i} \\
+A(y) \xi+B(y) u+O_{y}^{[3]}(\bar{\xi}, u) \\
y=\xi_{n}
\end{array}\right)+\left(\begin{array}{c}
0 \\
\vdots \\
0 \\
\sum_{i=1}^{n-1} \bar{c}_{i}^{n}(y) \xi_{i}
\end{array}\right)
\end{array}\right.
$$

Assuming $\phi_{y, n}^{[2]}(\bar{x})=0$, we have

and if we set

$$
\vartheta_{y, n}^{[1]}(\bar{x})=\bar{\vartheta}_{y, n}^{[1]}(\bar{x})
$$

$$
\vartheta_{y}^{[1]}(\bar{x})+\frac{\partial \phi_{y}^{[2]}(\bar{x})}{\partial x_{1}} b(y)=0
$$

then we obtain $\bar{\vartheta}_{y, 1}^{[1]}(\bar{x})=\ldots=\bar{\vartheta}_{y, n-1}^{[1]}(\bar{x})=0$.

According to the first homologic equation in (7),

$$
\gamma_{y}^{[2]}(\bar{x})+\Gamma_{y}^{[2]}(\bar{x})=A(y) \phi_{y}^{[2]}(\bar{x})+\tilde{\gamma}_{y}^{[2]}(\bar{x})
$$

we obtain

$$
\left\{\begin{array}{c}
\tilde{\gamma}_{y, 1}^{[2]}(\bar{x})=\gamma_{y, 1}^{[2]}(\bar{x})+\sum_{i=1}^{n-2}\left[\frac{\partial \phi_{y, 1}^{[2]}(\bar{x})}{\partial x_{i+1}} x_{i} \alpha_{i}(y)\right] \\
\tilde{\gamma}_{y, 2}^{[2]}(\bar{x})+\alpha_{1}(y) \phi_{y, 1}^{[2]}(\bar{x})=\gamma_{y, 2}^{[2]}(\bar{x}) \\
+\sum_{i=1}^{n-2}\left[\frac{\partial \phi_{y, 2}^{[2]}(\bar{x})}{\partial x_{i+1}} \alpha_{i}(y) x_{i}\right] \\
\vdots \\
\tilde{\gamma}_{y, n-1}^{[2]}(\bar{x})+\alpha_{n-2}(y) \phi_{y, n-2}^{[2]}(\bar{x})=\gamma_{y, n-1}^{[2]}(\bar{x}) \\
+\sum_{i=1}^{n-2}\left[\frac{\partial \phi_{y, n-1}^{[2]}(\bar{x})}{\partial x_{i+1}} \alpha_{i}(y) x_{i}\right] \\
\tilde{\gamma}_{y, n}^{[2]}(\bar{x})+\alpha_{n-1}(y) \phi_{y, n-1}^{[2]}(\bar{x})=\gamma_{y, n}^{[2]}(\bar{x})
\end{array}\right.
$$

From equation (15), the term $\phi_{y}^{[2]}(\bar{x})$ can be used to cancel all the quadratic terms form the second line 
to the last one, except for the terms $x_{1} \sum_{i=1}^{n-1} d_{i}^{j} x_{i}, j \in$ $[1, n]$, hence we obtain:

$$
\begin{aligned}
& \tilde{\gamma}_{y}^{[2]}(\bar{x})= \\
& \left(\sum_{j \geq i=1}^{n-1} d_{i}^{1}(y) x_{i} x_{j}, x_{1} \sum_{i=1}^{n-1} d_{i}^{2}(y) x_{i}, \cdots, x_{1} \sum_{i=1}^{n-1} d_{i}^{n}(y) x_{i}\right)^{T}
\end{aligned}
$$

Finally we have the normal form (14).

The following example is to illustrate the proposed normal form.

Example 2. (Example 1 continue) According to the above method, by simple calculation, we have

$$
\left\{\begin{array}{l}
z_{1}=x_{1}+2 x_{3} x_{1}^{2}+2 x_{3}^{3} x_{1} x_{2}+x_{3} x_{2}^{2} \\
z_{2}=x_{2}+x_{1} x_{2}+x_{3} x_{1}^{2} \\
z_{3}=x_{3}
\end{array}\right.
$$

with this diffeomorphism we have the following normal form:

$$
\left\{\begin{array}{c}
\dot{z}_{1}=\left(1+2 z_{3}^{4}\right) z_{1}^{2}+z_{3}^{2} z_{2}^{2}+z_{3} u \\
\quad+O_{y}^{[3]}\left(z_{1}, z_{2}, u\right) \\
\dot{z}_{2}=z_{3} z_{1}+\left(z_{3}-2 z_{3}^{2}\right) z_{1}^{2}-2 z_{3}^{4} z_{1} z_{2} \\
\quad+O_{y}^{[3]}\left(z_{1}, z_{2}, u\right) \\
\dot{z}_{3}=z_{3} z_{2}-z_{3} z_{1} z_{2}-z_{3}^{2} z_{1}^{2}+z_{3}^{2} z_{1} u \\
\quad+O_{y}^{[3]}\left(z_{1}, z_{2}, u\right) \\
y=z_{3}
\end{array}\right.
$$

\section{CHARACTERISTIC NUMBERS}

In order to simplify the calculation of diffeomorphism proposed before, a new method will be proposed in this section which permit us to determine the diffeomorphism (6) in a easer way, with which we need not to solve the homologic equation (7).

\subsection{Characteristic numbers for normal form with} drift dominant term

In order to simplify equation (7), we assume:

$$
\left\{\begin{aligned}
\phi_{y, i}^{[2]}(\bar{x}) & =x^{T} \phi_{y, i} x, \\
\bar{\gamma}_{y, i}^{[2]}(\bar{x}) & =x^{T} \bar{\gamma}_{y, i} x, \\
\gamma_{y, i}^{[2]}(\bar{x}) & =x^{T} \gamma_{y, i} x,
\end{aligned}\right.
$$

and

$$
\left\{\begin{array}{c}
\phi_{y, i}:=\left(\begin{array}{cccc}
\phi_{11}^{i}(y) & \cdots & \phi_{1, n-1}^{i}(y) & 0 \\
\vdots & \ddots & \vdots & \vdots \\
\phi_{1, n-1}^{i}(y) & \cdots & \phi_{n-1, n-1}^{i}(y) & 0 \\
0 & \cdots & 0 & 0
\end{array}\right), \\
\tilde{\gamma}_{y, i}:=\left(\begin{array}{cccc}
\tilde{\gamma}_{11}^{i}(y) & \cdots & \tilde{\gamma}_{1, n-1}^{i}(y) & 0 \\
\vdots & \ddots & \vdots & \vdots \\
\tilde{\gamma}_{1, n-1}^{i}(y) & \cdots & \tilde{\gamma}_{n-1, n-1}^{i}(y) & 0 \\
0 & \cdots & 0 & 0
\end{array}\right), \\
\gamma_{y, i}:= \\
\left.\begin{array}{cccc}
\gamma_{11}^{i}(y) & \cdots & \gamma_{1, n-1}^{i}(y) & 0 \\
\vdots & \ddots & \vdots & \vdots \\
\gamma_{1, n-1}^{i}(y) & \cdots & \gamma_{n-1, n-1}^{i}(y) & 0 \\
0 & \cdots & 0 & 0
\end{array}\right) .
\end{array}\right.
$$

We obtain $\Gamma_{y}^{[2]}(\bar{x})=\left[\Gamma_{y, 1}^{[2]}(\bar{x}), \ldots, \Gamma_{y, n}^{[2]}(\bar{x})\right]^{T}$ where $\Gamma_{y, i}^{[2]}(\bar{x})=x^{T} \Gamma_{y, i} x$ and

$$
\Gamma_{y, i}=\bar{A}^{T}(y) \phi_{y, i}+\phi_{y, i} \bar{A}(y),
$$

where

$$
\bar{A}(y):=\left(\begin{array}{ccccc}
0 & \ldots & 0 & 0 & 0 \\
\alpha_{1}(y) & \ldots & 0 & 0 & 0 \\
\vdots & \ddots & \vdots & \vdots & \vdots \\
0 & \ldots & \alpha_{n-2}(y) & 0 & 0 \\
0 & \ldots & 0 & 0 & 0
\end{array}\right) .
$$

Setting $A(y) \phi_{y}^{[2]}(\bar{x}):=x^{T} \bar{\phi}_{y} x$, for all $x$, homologic equation (7) can be written as follows:

$$
x^{T} \gamma_{y} x+x^{T} \Gamma_{y} x=x^{T} \bar{\phi}_{y} x+x^{T} \tilde{\gamma}_{y} x
$$

And we have

$$
\gamma_{y}+\Gamma_{y}=\bar{\phi}_{y}+\tilde{\gamma}_{y}
$$

if $\tilde{\gamma}_{y}=0$, we can cancel all the quadratic terms in system (4).

Because $A(y) \phi_{y}^{[2]}(\bar{x})=\left(\begin{array}{c}0 \\ \alpha_{1}(y) \phi_{y, 1}^{[2]}(\bar{x}) \\ \vdots \\ \alpha_{n-1}(y) \phi_{y, n-1}^{[2]}(\bar{x})\end{array}\right)$ if $\tilde{\gamma}_{y}^{[2]}=0$, then

$$
\begin{aligned}
& \left(\begin{array}{l}
\gamma_{y, 1} \\
\gamma_{y, 2} \\
\vdots \\
\gamma_{y, n}
\end{array}\right)+\bar{A}^{T}(y)\left(\begin{array}{c}
\phi_{y, 1} \\
\phi_{y, 2} \\
\vdots \\
\phi_{y, n}
\end{array}\right)+\left(\begin{array}{c}
\phi_{y, 1} \\
\phi_{y, 2} \\
\vdots \\
\phi_{y, n}
\end{array}\right) \bar{A}(y) \\
& =\left(0, \alpha_{1}(y) \phi_{y, 1}, \cdots, \alpha_{n-1}(y) \phi_{y, n-1}\right)^{T}
\end{aligned}
$$

we have

$$
\left\{\begin{array}{l}
\gamma_{y, 1}+\bar{A}^{T}(y) \phi_{y, 1}+\phi_{y, 1} \bar{A}(y)=0 \\
\alpha_{i}(y) \phi_{y, i}=\gamma_{y,{ }_{i+1}}+\bar{A}^{T}(y) \phi_{y,{ }_{i+1}}+\phi_{y,{ }_{i+1}} \bar{A}(y),
\end{array}\right.
$$

for $1 \leq i \leq n-1$.

Finally, by recurrence, we get

$$
\phi_{y, i}=\sum_{k=0}^{n-1-i}\left\{\frac{\sum_{j=0}^{k}\left[C_{j}^{k}\left(\bar{A}^{T}(y)\right)^{k-j} \gamma_{y, i+k+1} \bar{A}^{j}(y)\right]}{\prod_{m=0}^{k} \alpha_{i+m}}\right\}
$$

for $1 \leq i \leq n-1$, where $C_{j}^{k}$ denotes the combinatorial coefficient.

With this diffeomorphism, according to the following equality: $\vartheta_{y}^{[1]}(\bar{x})+\frac{\partial \phi_{y}^{[2]}(\bar{x})}{\partial x_{1}} b(y)=\bar{\vartheta}_{y}^{[1]}(\bar{x})$ we have $\vartheta_{y, n}^{[1]}(\bar{x})=\bar{\vartheta}_{y, n}^{[1]}(\bar{x})$. Setting

$$
\left\{\begin{array}{l}
\vartheta_{y, n}^{[1]}(\bar{x})=\sum_{j=1}^{n-1} c_{j}^{n}(y) x_{j}, \\
\bar{\vartheta}_{y, n}^{[1]}(\bar{x})=\sum_{j=1}^{n-1} \bar{c}_{j}^{n}(y) x_{j},
\end{array}\right.
$$

then $\bar{c}_{j}^{n}(y)=c_{j}^{n}(y)$. 
Because $\vartheta_{y}^{[1]}(\bar{x})+\frac{\partial \phi_{y}^{[2]}(\bar{x})}{\partial x_{1}} b(y)=\bar{\vartheta}_{y}^{[1]}(\bar{x})$, assuming

$$
\left\{\begin{array}{l}
\vartheta_{y, i}^{[1]}(\bar{x})=\sum_{j=1}^{n-1} c_{j}^{i}(y) x_{j}, \\
\bar{\vartheta}_{y, i}^{[1]}(\bar{x})=\sum_{j=1}^{n-1} \bar{c}_{j}^{i}(y) x_{j} .
\end{array}\right.
$$

then, since $\phi_{y, i}^{[2]}(\bar{x})=x^{T} \phi_{y, i} x$, we obtain:

$$
\left\{\begin{array}{l}
\bar{c}_{j}^{i}(y)=c_{j}^{i}(y)+2 b(y) \phi_{1, j}^{i}(y) \\
\bar{c}_{j}^{n}(y)=c_{j}^{n}(y)
\end{array}\right.
$$

for $1 \leq i, j \leq n-1$.

Definition 2. We define the characteristic matrix for system (4) as follows:

$$
M_{y}=\gamma_{y, 1}+\bar{A}^{T}(y) \phi_{y, 1}+\phi_{y, 1} \bar{A}(y)
$$

and

$$
C_{y}=\left(\begin{array}{cccc}
\bar{c}_{1}^{1}(y) & \cdots & \bar{c}_{n-1}^{1}(y) & 0 \\
\vdots & \ddots & \vdots & \vdots \\
\bar{c}_{1}^{n}(y) & \cdots & \bar{c}_{n-1}^{n}(y) & 0
\end{array}\right)
$$

where $M_{y}$ and $C_{y}$ are only function of $y$.

We are now able to set the following theorem.

Theorem 3. Normal form correspondent to drift dominant term of system (5) is as follows:

$$
\left\{\begin{array}{c}
\dot{\xi}=\xi^{T}\left(\begin{array}{c}
M_{y} \\
0 \\
\vdots \\
0
\end{array}\right) \xi+A(y) \xi+B(y) u \\
+C_{y} \xi u+O_{y}^{[3]}(\bar{\xi}, u) \\
y=\xi_{n}
\end{array}\right.
$$

where $M_{y}$ is defined in (22).

Remark 2. i) $M_{y}(i, j)$ in equation (24) depends on coefficients $h_{i j}(y)$ of equation (9) as follows:

$$
\left\{\begin{array}{l}
M_{y}(i, j)=M_{y}(j, i)=\frac{1}{2} h_{i j}(y), i<j \\
M_{y}(i, i)=h_{i i}(y)
\end{array}\right.
$$

ii) there are $3 n(n-1) / 2$ characteristic numbers in the normal form.

Example 3. (Example 1 continue) With the guide of the computation process proposed above, we have

$$
M_{y}=\gamma_{y, 1}+\bar{A}^{T}(y) \phi_{y, 1}+\phi_{y, 1} \bar{A}(y)=\left(\begin{array}{ccc}
1 & 0 & 0 \\
0 & x_{3}^{2} & 0 \\
0 & 0 & 0
\end{array}\right)
$$

and $C_{y}=\left(\begin{array}{ccc}-4 x_{3}^{2} & -2 x_{3}^{4} & 0 \\ -2 x_{3}^{2} & -x_{3} & 0 \\ x_{3}^{2} & 0 & 0\end{array}\right)$. Finally we get the same normal form correspondent to drift dominant term in (13).
4.2 Characteristic numbers for normal form with forced dominant term

With the same argument, in order to simplify equation (7), setting

$$
\left\{\begin{array}{l}
\vartheta_{y, n}^{[1]}(\bar{x})=\sum_{j=1}^{n-1} c_{j}^{n}(y) x_{j}, \\
\bar{\vartheta}_{y, n}^{[1]}(\bar{x})=\sum_{j=1}^{n-1} \bar{c}_{j}^{n}(y) x_{j},
\end{array}\right.
$$

and

$$
\bar{c}_{j}^{n}(y)=c_{j}^{n}(y) .
$$

Since $\vartheta_{y}^{[1]}(\bar{x})+\frac{\partial \phi_{y}^{[2]}(\bar{x})}{\partial x_{1}} b(y)=\bar{\vartheta}_{y}^{[1]}(\bar{x})$, and

$$
\phi_{y, i}^{[2]}(\bar{x})=x^{T}\left(\begin{array}{cccc}
\phi_{11}^{i}(y) & \cdots & \phi_{1, n-1}^{i}(y) & 0 \\
\vdots & \ddots & \vdots & \vdots \\
\phi_{1, n-1}^{i}(y) & \cdots & \phi_{n-1, n-1}^{i}(y) & 0 \\
0 & \cdots & 0 & 0
\end{array}\right) x,
$$

if we set $\phi_{1, j}^{i}(y)=-\frac{c_{j}^{i}(y)}{2 b(y)} 1$, we obtain $\bar{\vartheta}_{y, 1}^{[1]}(\bar{x})=\ldots=$ $\bar{\vartheta}_{y, n-1}^{[1]}(\bar{x})=0$. Then we have $\gamma_{y}+\Gamma_{y}=\bar{\phi}_{y}+\tilde{\gamma}_{y}$, which gives

$\left\{\begin{array}{l}\gamma_{y, 1}+\bar{A}(y) \phi_{y, 1}+\phi_{y, 1} \bar{A}(y)=\bar{\gamma}_{y, 1} \\ \bar{\gamma}_{y_{i+1}}+\alpha_{i}(y) \phi_{y, i}=\gamma_{y,,_{i+1}}+\bar{A}^{T}(y) \phi_{y,{ }_{i+1}}+\phi_{y, i+1} \bar{A}(y)\end{array}\right.$ for $1 \leq i \leq n-1$.

Defining

$$
\begin{aligned}
& \Upsilon_{y, i}=\left(\begin{array}{cccc}
\Upsilon_{11}^{i}(y) & \cdots & \Upsilon_{1, n-1}^{i}(y) & 0 \\
\vdots & \ddots & \vdots & \vdots \\
\Upsilon_{1, n-1}^{i}(y) & \cdots & \Upsilon_{n-1, n-1}^{i}(y) & 0 \\
0 & \cdots & 0 & 0
\end{array}\right)= \\
& \sum_{k=0}^{n-1-i}\left\{\frac{\sum_{j=0}^{k}\left[C_{j}^{k}\left(\bar{A}^{T}(y)\right)^{k-j}\left[\begin{array}{l}
\gamma_{y, i+k+1} \\
-\tilde{\gamma}_{y, i+k+1}
\end{array}\right] \bar{A}^{j}(y)\right]}{\prod_{m=0}^{k} \alpha_{i+m}}\right\}
\end{aligned}
$$

and

$$
\begin{aligned}
& \bar{\Upsilon}_{y, i}=\left(\begin{array}{cccc}
\bar{\Upsilon}_{11}^{i}(y) & \cdots & \bar{\Upsilon}_{1, n-1}^{i}(y) & 0 \\
\vdots & \ddots & \vdots & \vdots \\
\bar{\Upsilon}_{1, n-1}^{i}(y) & \cdots & \bar{\Upsilon}_{n-1, n-1}^{i}(y) & 0 \\
0 & \cdots & 0 & 0
\end{array}\right) \\
& =\sum_{k=0}^{n-1-i}\left\{\frac{\sum_{j=0}^{k}\left[C_{j}^{k}\left(\bar{A}^{T}(y)\right)^{k-j} \gamma_{y, i+k+1} \bar{A}^{j}(y)\right]}{\prod_{m=0}^{k} \alpha_{i+m}}\right\}
\end{aligned}
$$

Because $\phi_{1, j}^{i}(y)=-\frac{c_{j}^{i}(y)}{2 b(y)}, i, j \in[1, n-1]$, if we set

$$
\phi_{l, s}^{i}(y)=\bar{\Upsilon}_{l, s}^{i}(y), i \in[1, n-1], l, s \in[2, n-1]
$$

we have $\tilde{\gamma}_{l, s}^{i}=0, i \in[2, n], l, s \in[2, n-1]$.

Hence we get the following diffeomorphism:

$$
\phi_{y, i}:\left\{\begin{array}{l}
\phi_{1, j}^{i}(y)=-\frac{c_{j}^{i}(y)}{2 b(y)}, i, j \in[1, n-1] \\
\phi_{l, s}^{i}(y)=\bar{\Upsilon}_{l, s}^{i}(y), i \in[1, n-1], l, s \in[2, n-1]
\end{array}\right.
$$


Because we have $\tilde{\gamma}_{l, s}^{i}=0, i \in[2, n], l, s \in[2, n-1]$, then $\tilde{\gamma}_{1, j}^{i}, i \in[2, n], j \in[1, n-1]$ can be determined by the following equation:

$$
\phi_{1, j}^{i}(y)=-\frac{c_{j}^{i}(y)}{2 b(y)}=\Upsilon_{1, j}^{i}(y)
$$

and we note

$$
M_{y_{k}}(i, j)=M_{y_{k}}(j, i)=\left\{\begin{array}{l}
\tilde{\gamma}_{1, j}^{k}, i=1 \\
0, i \neq 1, n
\end{array},\right.
$$

for $1 \leq j \leq n-1$ and $2 \leq k \leq n$.

Finally because $\gamma_{y, 1}+\bar{A}(y) \phi_{y, 1}+\phi_{y, 1} \bar{A}(y)=\tilde{\gamma}_{y, 1}$, we obtain:

$$
M_{y, 1}(i, j)=M_{y, 1}(j, i)=\tilde{\gamma}_{i, j}^{1}, i<j
$$

Then we can give the following theorem.

Theorem 4. Normal form correspondent to forced dominant term of system (5) is as follows:

$$
\left\{\begin{array}{c}
\dot{\xi}=\xi^{T}\left(\begin{array}{c}
M_{y, 1} \\
M_{y, 2} \\
\vdots \\
M_{y, n}
\end{array}\right) \xi+\left(\begin{array}{c}
0 \\
\vdots \\
0 \\
\sum_{i=1}^{n-1} \bar{c}_{i}^{n}(y) \xi_{i}
\end{array}\right) u \\
+A(y) \xi+B(y) u+O_{y}^{[3]}(\bar{\xi}, u) \\
y=\xi_{n}
\end{array}\right.
$$

where $\bar{c}_{i}^{n}$ and $M_{y, i}$ are defined by equations (25), (28) and (29).

Remark 3. The number of the free coefficients is $3 n(n-1) / 2$.

Example 4. (Example 1 continue) Following the proposed calculation procedure, we can obtain

$$
\phi_{1}(y)=\left[\begin{array}{ccc}
2 x_{3} & x_{3}^{3} & 0 \\
x_{3}^{3} & x_{3} & 0 \\
0 & 0 & 0
\end{array}\right], \phi_{2}(y)=\left[\begin{array}{ccc}
x_{3} & \frac{1}{2} & 0 \\
\frac{1}{2} & 0 & 0 \\
0 & 0 & 0
\end{array}\right], \phi_{3}(y)=0
$$

and the following matrix $M_{y}$ :

$$
\left\{\begin{aligned}
M_{y, 1}= & \left(\begin{array}{ccc}
1+2 x_{3}^{4} & 0 & 0 \\
0 & x_{3}^{2} & 0 \\
0 & 0 & 0
\end{array}\right), M_{y, 2}=\left(\begin{array}{ccc}
x_{3}-2 x_{3}^{2} & -x_{3}^{4} & 0 \\
-x_{3}^{4} & 0 & 0 \\
0 & 0 & 0
\end{array}\right), \\
M_{y, 3}= & \left(\begin{array}{ccc}
-x_{3}^{2} & -\frac{1}{2} x_{3} & 0 \\
-\frac{1}{2} x_{3} & 0 & 0 \\
0 & 0 & 0
\end{array}\right)
\end{aligned}\right.
$$

which yields the same normal form (18).

\section{CONCLUSION}

This paper is devoted to study the quadratic observability normal form parameterized by its output. Above all, two homologic equations were given in order to guarantee the equivalence of quadratic transformation. After that, we have studied two normal forms respectively correspondent to the drift dominant term and forced dominant term. Both representations are equivalent. In order to simplify the calculation, we proposed to apply quadratic terms' characteristic matrix for these two normal forms.

\section{REFERENCES}

Boutat D., Zheng G., Barbot J.P. and Hammouri H. (2006), "Observer Error Linearization Multi-Output Depending", In Proc. of IEEE CDC, 2006.

Boutat-Baddas L., Boutat D., Barbot J.P. and Tauleigne R.(2001), "Quadratic Observability normal form", In Proc. of IEEE CDC, 2001.

Chabraoui S., Boutat D., Boutat-Baddas L. and Barbot J.P. (2003), "Observability quadratic characteristic numbers", In Proc. of IEEE CDC, 2003.

Poincaré H. (1899), "Les Méthodes nouvelles de la mécanique céleste", Gauthier Villard, 1899 Réedition 1987, B.bibliothèque scientifique $A$. Blanchard.

Krener A.J., (1984), "Approximate linearization by state feedback and coordinate change", Systems and Control Letter, 5, 181-185.

Krener A.J., Karahan S., Hubbard M. and Frezza R. (1987), "Higher order linear approximations to nonlinear control systems ", In Proc. of IEEE CDC, $198 \%$.

Krener A.J., Karahan S. and Hubbard M., (1988), "Approximate normal forms of nonlinear systems", In Proc. of IEEE CDC, 1988.

Kang W. and Krener A.J. (1992), "Extended quadratic controller normal form and dynamic state feedback linearization of non linear systems", SIAM J. Control and Optimization, Vol 30, No 6, pp 1319-1337, 1992.

Zheng G., Boutat D. and Barbot J.P. (2005), "Output Dependent Observability Normal Form", In Proc. of IEEE CDC, 2005. 